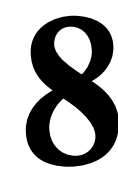

\title{
PEDAGOGÍA DE LA MUERTE MEDIANTE APRENDIZAJE SERVICIO
}

(PEDAGOGY OF DEATH BY SERVICE LEARNING)

Pablo Rodríguez Herrero y Agustín de la Herrán Gascón Universidad Autónoma de Madrid

Mar Cortina Selva

Florida Universitaria, Valencia

DOI: $10.5944 / e d u c X X 1.18 .1 .12317$

Cómo referenciar este artículo/How to reference this article:

Rodríguez Herrero, P.; de la Herrán Gascón, A. y Cortina Selva, M. (2015). Pedagogía de la muerte mediante aprendizaje de servicio. Educación XX1, 18(1), 189-212. doi: 10.5944/educXX1.18.1.12317

Rodríguez Herrero, P.; de la Herrán Gascón, A. y Cortina Selva, M. (2015). Pedagogy of death by service learning. Educación XX1, 18(1), 189-212. doi: 10.5944/educXX1.18.1.12317

\section{RESUMEN}

La inclusión de la muerte en la educación como elemento formativo es todavía un reto emergente. Su introducción didáctica podría, en nuestra opinión, contribuir al desarrollo de una educación que incluya «temas radicales o perennes», entendiendo por tales los que, como el que se presenta, no se demandan, no están expresamente integrados en los currícula ni en los proyectos pedagógicos de los centros y son esenciales para la formación. De acuerdo con ello urge especialmente generar metodologías didácticas que puedan favorecer su tratamiento educativo. El aprendizaje servicio (AS) puede favorecer aspectos tan relevantes en la educación actual como la formación ciudadana, la acción solidaria o la responsabilidad social. Su vinculación con la acción social entronca con una de las posibles finalidades de la Pedagogía de la Muerte, que es formar ciudadanos que consideren la finitud como condición humana desde la cual orientar valores vitales para la mejora social. En este artículo se plantea el AS como propuesta metodológica desde la que es posible desarrollar la Pedagogía de la Muerte, describiéndose varias actividades didácticas para las distintas etapas educativas. En las conclusiones se valora el AS como propuesta metodológica de una posible Pedagogía de la Muerte, destacándose la necesidad de una formación del profesorado de calidad. 


\section{PALABRAS CLAVE}

Pedagogía de la muerte, aprendizaje profesional, formación de profesores.

\section{ABSTRACT}

The inclusion of death in education as a formative element is still an emerging challenge. Its didactic introduction, in our opinion, could contribute to the development of education that includes "radical or perennial issues», defined as those that, like the present case, not are required, nor explicitly integrated into curriculum or teaching projects and are essential for human formation. Accordingly, it is particularly urgent to generate teaching methodologies that can foster their educational treatment. Service Learning (SL), can support important aspects in education today such as civic education, common action and social responsibility. Its connection to social action is connected with one of the possible aims of the Pedagogy of Death, which is to train citizens to consider the human condition of finitude as a guiding value that is vital for social improvement. This article presents the SL as a methodology proposal from which it is possible to develop the Pedagogy of Death, describing various educational activities for the different stages of education. The conclusions assess the SL as a possible methodological approach of Pedagogy of Death, highlighting the need for quality teacher training ahead of their proper use.

\section{KEY WORDS}

Death, education, apprenticeship, teacher training.

"Cada hombre, desde niño, debe ser conocido, reconocido y tratado como miembro necesario y esencial de la humanidad»

Friedrich Fröbel (1782-1852)

\section{INTRODUCCIÓN}

Todavía existe en la cultura occidental lo que Geoffrey Gorer denominó «pornografía de la muerte» (1955), si cabe en ciertos aspectos mayor que hace casi seis décadas. Un progreso desprovisto de humanidad y pedagógicamente desorientado (Herrán, 2008) ha favorecido que se desarrolle un tabú hacia la muerte, nutrido por medios de comunicación o por agentes que, en cierta manera, tratan de negar al individuo su encuentro educativo 
con la muerte. El contacto actual entre el ser humano y la muerte es generalmente ficticio y fundamentado en el morbo, despojado de su natural y profunda relación dialéctica que puede, a nuestro entender, contribuir a la formación personal y social. La muerte incluye la conciencia de la propia finitud y la elaboración de significados en torno a esta mortalidad. La educación puede, desde paulatinos apoyos pedagógicos, contribuir a la evolución formativa de los significados que docentes y discentes otorgan a la muerte en general y a la propia en particular.

Aceptar la negación individual en pro de la evolución y la mejora de la humanidad no es fácil empresa, si consideramos las raíces del tabú a la muerte, más hondas y profundas que las del tabú al sexo, que comienza a ser considerado en las últimas décadas en la sociedad y en la educación: «Un rasgo significativo de las sociedades más industrializadas es que la muerte ha ocupado en ellas el lugar de la sexualidad en tanto que el mayor de los tabús» (Ariés, 2000, p. 289). Aceptar la muerte como fenómeno natural y esencial al ser humano constituye un difícil camino en el que se hace constante el enfrentamiento con la supervivencia individual. La iniciativa parte, como decía Nietzsche, de la valentía (1980, p. 17): «Toda conquista, todo paso adelante en el conocimiento es consecuencia del valor». La recompensa es, sin embargo, reconfortante: primero, sentirse parte de la humanidad y segundo, poder contribuir más conscientemente a su mejora y transformación. Decíamos, empero, que el diálogo entre el individuo y el hombre, como especie en evolución, es complicado, como nos dan a entender grandes literatos de la historia a través de sus obras. El joven Werther de Goethe luchará en sus últimos momentos en la compaginación de su pensamiento y su pasión. Igual le pasaría a Ivan Illich, de Tolstoi, quien en su agonía sufrirá a intervalos el duelo entre la impotencia de su corazón y la lógica de su racionalidad.

A nuestro entender la educación no puede separarse del ser humano, y por tanto no puede estar desvinculada de lo que al hombre caracteriza y preocupa. En un estudio realizado por Bowie (2000) se investigaron las actitudes hacia la muerte en el alumnado de educación primaria del Reino Unido y se encontró que un $73 \%$ de los alumnos manifestaba pensar en la muerte. Los niños, desde una edad muy temprana, comienzan a integrar la muerte en sus juegos y en sus conversaciones. Si la muerte es parte de la vida de los niños, adolescentes y adultos, y a todos ellos les importa esencialmente, ¿por qué no incluirla expresamente en la educación? (Herrán, González, Navarro, Freire y Bravo, 2000). La enseñanza y los centros docentes deben ocuparse, sobre todo, de lo que más importa al ser humano.

La muerte tiene un potencial formativo inherente para la educación en valores, como se ha estudiado en investigaciones recientes (Rodríguez 
Herrero, Herrán y Cortina, 2013), por lo que es preciso que los educadores conozcan metodologías didácticas desde las que introducir el tema en la educación. Una metodología destacada con esta orientación es el «aprendizaje servicio». A partir de los años 60 del pasado siglo se comienza a denominar "aprendizaje servicio» (AS) a una metodología didáctica que relaciona curriculum y servicio social a la comunidad para el aprendizaje. El AS combina contenidos y objetivos curriculares con experiencias directas o indirectas de acción social. La muerte, como ámbito perenne del ser humano, está presente en innumerables contextos de la sociedad, por lo que la experiencia del alumnado en estos marcos de acción social, planificados, ejecutados y evaluados desde un enfoque formativo, pueden incluirse en una posible Pedagogía de la Muerte.

\section{PEDAGOGía dE LA MUERTE}

La muerte es un ámbito de estudio relativamente aceptado, desde perspectivas diferentes, en disciplinas como la Psicología, Biología, Sociología o Antropología. Sin embargo, hasta hace pocos años no lo era en Pedagogía. Si aquellas disciplinas han centrado su atención en una lectura de la muerte como pérdida, sufrimiento y factor cultural, la Pedagogía puede y debe liderar la normalización educativa y social de la muerte como posible constructo desde el que promover una sociedad más humana, solidaria, culta y madura. Por ello una atención científica de la Pedagogía hacia la muerte constituiría, no solo un complemento a la investigación de la muerte en las disciplinas señaladas, sino también un avance cualitativo sustancial. En los últimos años y desde España se vienen desarrollando iniciativas pioneras en Pedagogía de la Muerte (Cortina, 2010; Rodríguez Herrero, 2012; Rodríguez Herrero, Herrán y Cortina, en prensa; Rodríguez Herrero, Herrán e Izuzquiza, 2013), que fundamenta, investiga y propone algo más que un cambio conceptual: «del tabú al imperativo educativo» (Herrán, González, Navarro, Bravo y Freire, 2000, 2001).

Este trabajo se relaciona más con las contribuciones que asocian educación para la muerte y educación de la conciencia. Por conciencia entendemos, con Torre (2006), «atributo más específico y relevante del ser humano en el mundo que conocemos, consistente en volver sobre sí y sobre las cosas para retornar de nuevo sobre nosotros con nuevos significados, en forma de espiral ascendente» (p. 143) o «lo que nos permite darnos cuenta de nosotros y de los demás» (Damasio, 2010). Puede ser un constructo relevante y fértil para la educación (Herrán, 1998, 2005, 2006; Cabrera, 2011) y es coherente con la inclusión de la muerte en la formación: «La Educación para la Muerte podría ser uno de los caminos para conectar a la educación ordinaria con la Educación para la Evolución Humana (...) consideramos 
que la Educación para la Muerte es una rama importante del árbol que nos interesa, la Educación de la Conciencia» (Herrán y Cortina, 2006, pp. 65-66; Herrán, 2014). Este enfoque está centrado en el valor de la muerte como ámbito formativo perenne. Comprende tanto acciones pedagógicas preventivas - independientes de una pérdida significativa - y paliativas - orientadas a la formación e intervención pedagógica individual o colectiva desde la tutoría con la metodología del acompañamiento educativo (Herrán y Cortina, 2008) - . Ambas perspectivas tienen por objeto la formación y la madurez, desde la convicción de que la interiorización a través de la didáctica orienta la actitud hacia el sufrimiento (Dürkheim, 1996, p. 221): «El sufrimiento atormenta o el sufrimiento hace madurar según sea la actitud del hombre que sufre».

La Pedagogía de la Muerte pretende una formación pedagógica específica del profesorado de todos los alumnos posibles, desde la educación infantil hasta la educación superior y de adultos, contemplando también a aquellos alumnos que pueden presentar necesidades específicas de apoyo educativo (por ejemplo, Rodríguez Herrero, 2012; Rodríguez Herrero et al., 2013) o que proceden de distintos países. Más allá de pertenecer a una cultura determinada, la muerte es un elemento transcultural y como tal puede considerarse dentro de una «educación para la universalidad» (Herrán y Muñoz, 2002), entendida desde una aproximación educativa como un ideal formativo que no es patrimonio de ninguna cultura sino de todas ellas, y que mira a promoverlas más allá de sus particularidades exclusivas (Savater, 1997). En una sociedad generalmente mercantilista y proyectada hacia la utilidad, la Pedagogía de la Muerte puede contribuir a la redefinición de valores vitales que orientan las acciones humanas, especialmente en aquellas etapas educativas en las que el tecnicismo está más presente.

Una posible introducción de la muerte en la educación exige del profesorado una formación pedagógica profunda y concreta (Herrán, 2014). Es en la educación de temas radicales o perennes donde se vislumbra la calidad del docente como maestro, su madurez y evolución personal. Ya decía Montaigne (2003, p. 108) que «Para juzgar de las cosas grandes y elevadas, es menester alma igual». O Nietzsche (1984, p. 134) que «La necesidad se considera como causa de la formación, pero en realidad es muchas veces efecto de lo que se ha formado". La formación inicial del profesorado o de los educadores ha de ir más allá de lo superficial para alcanzar metas educativas elevadas; y cuando se toma conciencia de ellas asoma también su valor formativo.

Señalábamos anteriormente el AS como una metodología didáctica interesante para incluir la muerte en la acción educativa. En los últimos años se vienen proponiendo distintos recursos y propuestas metodológicas para 
introducir el tema de la muerte en la educación (véase, por ejemplo, Herrán y Cortina, 2006; Herrán et al., 2000): a) cine-video fórum (Cortina, 2010); b) literatura infantil y juvenil; c) música; d) muertes parciales; e) humor; f) diálogo sobre la duda; g) juegos de roles; h) pequeñas investigaciones; i) proyectos didácticos; j) entrevistas con expertos; k) talleres; l) técnicas de aprendizaje cooperativo; etc. Nuestro propósito, a través de este trabajo, es plantear el AS como metodología didáctica susceptible de formar parte de una posible Pedagogía de la Muerte.

\section{APRENDIZAJE SERVICIO}

El término AS surge a finales de los años 60 del siglo pasado con las aportaciones de R. Sigman y W. Ramsey (Titlebaum, Williamson, Daprano, Baer y Brahler, 2004). Se nutre de las ideas de pedagogos como Comenius, Rousseau, Decroly, Makarenko, Sujomlinsky o Dewey. Comenius (15921670) ya destacaba en su Didáctica Magna la relevancia de transferir los aprendizajes a la vida cotidiana. Rousseau (1712-1778) destacaría en Emilio o de la educación la importancia que para el desarrollo del niño tiene el aprendizaje activo y la observación directa, al igual que lo haría Decroly (1871-1932) más tarde. Tesis parecidas defenderían los pedagogos soviéticos Sujomlinsky (1918-1970) y el más conocido Makarenko (1888-1939), quien en obras como Poema pedagógico o Colectividad y educación afirmaría la experiencia como principio básico de la enseñanza. Las ideas de Dewey (1859-1952), por su parte, serán fundamentales para el surgimiento del AS. De hecho, en 1921 se crearía un programa comunitario estudiantil en Antach College fruto de las aportaciones de Dewey (Tapia, 2008).

Puig, Batlle, Bosch y Palos (2006, p. 20) lo definen como: «una propuesta educativa que combina procesos de aprendizaje y de servicio a la comunidad en un solo proyecto bien articulado en el que los participantes se formen al trabajar sobre necesidades reales del entorno con el objetivo de mejorarlo». Tapia, por su parte, arguye que es «el servicio solidario protagonizado por los estudiantes, destinado a cubrir necesidades reales de una comunidad, planificado en forma integrada con el currículo, en función del aprendizaje de los estudiantes» (Tapia, 2004, p. 15).

Si bien las definiciones propuestas por los autores recogen los principales rasgos del AS, consideramos que es importante matizar algunos aspectos: a) que el aprendizaje no tiene por qué estar vinculado a la formación. Existen aprendizajes muy poco formativos, e incluso desaprendizajes significativos que permiten evolucionar al niño o al adolescente (Herrán, 2005); y b) que el servicio a la comunidad, a nuestro entender, no ha de dirigirse al progreso local o del entorno próximo sino al desarrollo de la humanidad, 
conscientes de que la mejora de la comunidad forma parte de la contribución a toda la humanidad. Pudiera decirse de esta matización que no varía los elementos del AS. Sin embargo, creemos que la acción social no es la misma, desde sus fundamentos y finalidades, si situamos su objeto en la mejora de la humanidad más allá de limitarlo al desarrollo local.

Conceptuamos el AS como una metodología pedagógica activa que integra actividades de formación complejas para la evolución personal y de la humanidad, desarrolladas tanto en el contexto escolar como en el servicio a otros entornos de la comunidad. Combina procesos formativos en el contexto educativo con el servicio social a la comunidad en proyectos globales que favorezcan la participación del alumnado en su entorno. Requiere de una gran coordinación entre las instituciones participantes, concretándose en una primera fase de preparación para después ejecutar y evaluar el proyecto a partir del cual se desarrolla. Como metodología didáctica, presenta las siguientes características: a) se aplica en ámbitos educativos formales y no formales (Puig et al., 2006); b) parte de contenidos y objetivos contemplados en el currículum; c) se fundamenta en principios como la formación activa y basada en la experiencia; d) requiere un trabajo en red que coordine las instituciones (Puig et al., 2006); e) integra el servicio a la comunidad, como representante de la humanidad y para su desarrollo; f) precisa de una gran coordinación entre los profesionales de un centro educativo debido a la posibilidad de que las actividades de AS contengan elementos interdisciplinares o transdisciplinares; g) favorece el desarrollo de las competencias básicas y de la educación en valores; h) facilita la adquisición de recursos resilientes en el alumnado (Tapia, 2004).

Como metodología didáctica compleja, el AS integra diversas fases que los educadores pueden tener presente: planificación, ejecución y evaluación. Destacamos a continuación algunas preguntas orientativas de cada etapa:

a) Planificación: ¿Qué elementos curriculares se quieren desarrollar? (objetivos, contenidos, competencias básicas). Si algunos de estos elementos no están contemplados en el Proyecto Educativo de Centro (como pudiera ser la muerte, o la propia metodología AS), ¿cómo podemos integrarlo en el currículum? ¿Cómo se tienen que coordinar los profesionales del centro educativo? (maestros o profesores, servicio o departamento de orientación, equipo directivo...). ¿Qué formación necesitan los profesionales que van a participar en la metodología basada en el AS? ¿Desde qué áreas se van a desarrollar las actividades? ¿Contienen las actuaciones planificadas elementos interdisciplinares o transdisciplinares? ¿Qué instituciones externas al centro educativo pueden participar en el AS? ¿Cómo se coordina el centro educativo con los diversos 
contextos en los que va a participar el alumnado a través de la acción social? ¿Cuál debe ser la participación de las familias en el desarrollo de las actividades planificadas desde el AS?

b) Ejecución: ¿Cuáles son los tiempos de participación del alumnado tanto en el contexto escolar como en otros entornos? ¿Cómo se da coherencia a la acción social directa y al trabajo en el aula? ¿Cuál es la respuesta y la actitud de las instituciones externas al centro educativo? ¿Se favorece la reflexión en el proceso de acción directa? ¿Cómo se responde a las necesidades surgidas en el alumnado?

c) Evaluación: ¿Se facilita la reflexión posterior para la formación? ¿Cómo se ha llevado a cabo la coordinación entre los distintos agentes que han participado? ¿Qué mejoras pueden derivarse de la experiencia a nivel didáctico y de organización? ¿Se favorece la (auto) evaluación crítica y constructiva? ¿Qué participación tienen las instituciones externas al centro que han participado en el AS en la evaluación de las experiencias? ¿Se crea un clima favorable para la indagación posterior en los aprendizajes y desaprendizajes (eliminación de prejuicios, creencias, estereotipos, etc.) del alumnado?

Uno de los aspectos básicos descritos es la vinculación que el AS tiene con el currículum de los centros educativos, y por lo tanto también con los proyectos educativos.

\section{ACTIVIDADES DE APRENDIZAJE SERVICIO INTERPRETADAS DESDE LA PEDAGOGÍA DE LA MUERTE}

Previamente a la exposición de propuestas didácticas de Pedagogía de la Muerte a través del AS analizamos varias experiencias reales desarrolladas con esta metodología que pueden interpretarse como desarrollos de Pedagogía de la Muerte, si bien no la contemplan explícitamente en sus objetivos. Esto es congruente si por muerte se amplía la noción tradicionalmente manejada por la Psicología (pérdida, sufrimiento, duelo), y se accede, además, a otras conceptuaciones pedagógicas que facilitan su accesibilidad curricular y metodológica: parte de la vida, muerte parcial, fase de ciclo vital, muerte continua, presencia de quienes no están, tema radical o transversal de transversales, pérdida del ego, muerte total, lectura transcultural, necesidad para la evolución, etc. (Herrán y Cortina, 2006).

a) La ecoauditoría en el aula (experiencia descrita en Rubio y Arroyes, 2010). Desarrolla la lectura de muerte comprendida como fenómeno que atraviesa un tema transversal clásico: la educación me- 
dioambiental, desde la perspectiva del deterioro (muerte potencial y en proceso) del entorno. El proyecto comprende varias actividades que desarrollan en colaboración el Centro Medioambiental L'Arrel, el Ayuntamiento de Sant Joan Despí y varias escuelas de la población catalana. El objetivo del proyecto es fomentar en el alumnado que participa el respeto hacia el medio ambiente y la responsabilidad con su cuidado. Propone que los alumnos participen de forma activa en la iniciativa de acciones que repercutan en la sostenibilidad y la evitación de su destrucción progresiva. El proyecto consta de varias fases: primero, la presentación a las escuelas de la localidad por técnicos del centro medioambiental y del ayuntamiento, para después abordar el trabajo directo con los alumnos con actividades de sensibilización y organización, investigación y diagnóstico, comunicación y plan de acción, realización de la propuesta, evaluación y finalmente la Fiesta Sostenible. En la fase de sensibilización se busca la interiorización de valores relacionados con el consumo responsable y con el respeto hacia las generaciones futuras a través de la sostenibilidad. Se intenta, por tanto, generar responsabilidad diacrónica en el alumnado. En las actividades de investigación y diagnóstico los alumnos han de analizar la situación medioambiental del centro y estudiar acciones de mejora. En relación, por ejemplo, a los residuos, se trabaja en torno a preguntas como: «averiguad cuáles son los tipos de residuos» o "haced el experimento de recoger y analizar los tipos de residuos que se generan en la escuela a la hora del recreo». Ya en la fase de planificación y plan de acción, y de acuerdo con las conclusiones generadas por los propios alumnos en la investigación, estos diseñan un plan de acción y transformación en el marco del grupoaula. En referencia de nuevo al tema de los residuos, se determinó promover la recogida de residuos y utilizar los contenedores. Posteriormente se llevan a cabo las acciones previstas por el alumnado con el apoyo de los técnicos del centro medioambiental y del ayuntamiento. Después de haber realizado el proyecto se aborda una sesión en el aula de evaluación, creando un contexto en el que analizar los aprendizajes desarrollados a través del proyecto. Se culmina con una Fiesta Sostenible reuniendo a las escuelas que han participado en el proyecto, a las familias y a otros agentes que han participado en el mismo. Los contenidos desarrollados, siguiendo a los autores que describen la experiencia, tienen relación con áreas curriculares como Conocimiento del Medio Natural, Social y Cultural o Matemáticas, así como con la educación en valores como el respeto al medioambiente y la sostenibilidad. En nuestra opinión, muchos de los objetivos planteados en este proyecto son compartidos con la Pedagogía de la Muerte. El respeto al medio ambiente, 
el ciclo vital, la conciencia de responsabilidad para con las futuras generaciones, y por tanto la responsabilidad socio-histórica son planteamientos educativos que pueden formar parte de la introducción didáctica de la muerte a través del trabajo en la naturaleza. Una forma de incluir más abiertamente el tema de la muerte en este proyecto puede consistir en integrar en las fases de sensibilización o reflexión lecturas o películas relacionadas con el ciclo de la vida, adaptadas a la edad y al desarrollo evolutivo de los alumnos.

d) Todos somos meninos (experiencia descrita en Rubio y Escardíbul, 2010). Desarrolla la lectura de muerte comprendida como efecto trágico y real de la desigualdad social. Este proyecto lo desarrollan en colaboración la Fundación Catalana l'Esplai y el Movimiento Nacional de Meninos e Meninas da Rua de Brasil. Consiste en la cooperación entre las dos entidades con el objetivo de que, a través de la educación no formal, se fomente la solidaridad mediante una experiencia de Aprendizaje Servicio. La actividad central del proyecto es la participación de jóvenes españoles en el Encontro Nacional de Meninos e Meninas da Rua celebrado periódicamente en Brasil, y consta de tres fases principales: la preparación previa, el viaje y la valoración posterior al viaje. Los jóvenes que participan en las actividades son adolescentes de entre 15 y 17 años. El alumnado que viaja a Brasil participa allí activamente en los talleres o las charlas que se realizan en el marco del Encontro. Los jóvenes conocen, así, la realidad de los niños y niñas en situaciones de desventaja social que viven en Brasil. Una fase muy significativa para el aprendizaje de los jóvenes es la valoración que realizan después del viaje a través de varios espacios y contextos. El grupo se reúne para analizar la experiencia y para expresar verbalmente todos los sentimientos que se han generado. Además, los jóvenes realizan una difusión de las actividades que han llevado a cabo a través de la participación en charlas o exposiciones. Los adolescentes que han participado en las actividades se convierten de tal forma en sensibilizadores de la sociedad, en agentes que promueven la acción social y la transformación. Los contenidos de aprendizaje que se desarrollan en las actividades son, siguiendo a Rubio y Escardíbul (2010): situación geográfica, social, política y económica de Brasil; aspectos culturales del país; el conocimiento de la realidad social de los niños y las niñas en situación de desventaja de Brasil; nociones de la lengua portuguesa; habilidades comunicativas; los derechos de los niños; o habilidades para la organización, funcionamiento y dinámica de la estancia de los jóvenes en Brasil. El servicio es mutuo, al acoger los niños y niñas del Encontro a los representantes de la Fundación. La Pedagogía de la Muerte en este contexto 
tiene, desde una perspectiva crítica (Mantegazza, 2006), un planteamiento que podemos denominar reivindicativo y que promueve el desarrollo de generaciones conscientes de las desigualdades que se producen en el mundo. Consideramos que, a través de experiencias solidarias como las realizadas en el proyecto Todos somos meninos, se puede favorecer la reflexión sobre la injusticia de muchas muertes y sobre la actuación de la sociedad en relación a las mismas. Lecturas que se acerquen a los intereses de los adolescentes y que toquen temas relacionados pueden complementar la formación que adquieren los jóvenes que participan en estas experiencias. Sugerimos en este sentido, por ejemplo, libros como Los juegos del hambre (Collins, 2009) o documentales como Noche y niebla (Resnais, 1955), que exponen de forma explícita el sinsentido de muertes ignoradas por la sociedad.

e) Recuperación de la historia local en el Ins. Agrotécnico San José Obrero-San Justo-Entre Ríos (experiencia descrita en Tapia, 2004): Desarrolla la lectura de muerte comprendida como presencia enriquecedora y orientadora de quienes murieron. La experiencia que se describe tuvo lugar en el Instituto Agrotécnico San José Obrero de la provincia argentina de Entre Ríos. La localidad carecía de museos o archivos de la historia de la ciudad y de la población que hasta entonces la ha habitado. En el instituto se pone en marcha un proyecto interdisciplinario de investigación y que abarcaba áreas curriculares diversas como Lengua, Historia, Geografía, Formación Ética y Ciudadana, y Matemáticas. Fruto de la reunión de los datos que investigaron los alumnos se crea en el año 2000 el «Museo de la Colonia». La experiencia, a nuestro juicio, podría formar de una posible Pedagogía de la Muerte que contemplara el diálogo intergeneracional y la consideración hacia personas que en un pasado contribuyeron al desarrollo local. En una sociedad marcada aparentemente por la quietud y orientada hacia el bienestar personal, experiencias de innovación educativa como la descrita pueden devolver a las nuevas generaciones la responsabilidad socio-histórica y la identificación con el ser humano como motor de cambio y de mejora social.

Estas experiencias tienen por denominador común mostrar que, a través del servicio solidario, los alumnos son capaces de despegarse de una realidad cada vez más virtual creciendo y madurando con acciones sociales cooperativas que favorecen su conocimiento y formación integral. Incluir la muerte en sus contenidos desde una vertiente didáctica y formativa ampliaría, en nuestra opinión, el horizonte pedagógico de dichas experiencias, la complejidad y por tanto la potencial profundización educativa del AS. 


\section{PROPUESTAS DIDÁCTICAS DE PEDAGOGÍA dE LA MUERTE DESDE EL APRENDIZAJE SERVICIO}

A continuación se muestran algunas propuestas didácticas de Pedagogía de la Muerte a través del AS para distintas etapas educativas:

Primera actividad: "Reforestación de un bosque»

Etapa: Infantil, Primaria o Secundaria (adaptable).

Objetivos:

- Tomar conciencia de la limitación de los recursos naturales y de la importancia de la acción del hombre en su preservación.

- Conocer el ciclo vital de las especies vegetales y de diferentes animales que conforman un espacio natural.

- Prevenir acciones abusivas y destructivas hacia la naturaleza.

Contenidos:

- Especies vegetales y animales.

- Ciclo vital.

- Reforestación.

Contribución a las competencias básicas:

- Competencia social y ciudadana.

- Autonomía e iniciativa personal.

- Competencia en el conocimiento y en la interacción con el mundo físico.

Áreas curriculares vinculadas:

- Conocimiento de sí mismo y autonomía personal (Infantil).

- Conocimiento del entorno (Infantil).

- Conocimiento del medio natural, social y cultural (Primaria). 
- Ciencias de la naturaleza (Secundaria).

- Educación ético-cívica (Secundaria).

- Educación para la ciudadanía y los derechos humanos (Primaria y Secundaria).

Sugerencias para su realización: Participar activamente en la naturaleza y en el ciclo vital de las especies vegetales puede ser un aprendizaje relevante de una posible Pedagogía de la Muerte. Se puede aprovechar la actividad para reflexionar, en función de la etapa y adaptado a sus necesidades, sobre el ciclo vital en el ser humano y su relación con la naturaleza.

Segunda actividad: «Reciclaje de basuras»

Etapa: Infantil, Primaria o Secundaria (adaptable).

Objetivos:

- Conocer qué se hace con la basura al tirarla, como expresión simbólica del desecho o de lo inutilizable.

- Tomar conciencia de la importancia del reciclaje para la conservación de los bienes naturales.

- Participar a través de la experiencia en el trabajo de un basurero para prevenir o eliminar prejuicios o estereotipos.

Contenidos:

- Formas de reciclaje.

- Tipos de basuras.

- Funcionamiento de los basureros.

Contribución a las competencias básicas:

- Competencia social y ciudadana.

- Competencia en el conocimiento y en la interacción con el mundo físico. 
Áreas curriculares vinculadas:

- Conocimiento de sí mismo y autonomía personal (Infantil).

- Conocimiento del entorno (Infantil).

- Conocimiento del medio natural, social y cultural (Primaria).

- Ciencias de la naturaleza (Secundaria).

- Educación ético-cívica (Secundaria).

- Educación para la ciudadanía y los derechos humanos (Primaria y Secundaria).

Sugerencias para su realización: La actividad puede incluir el trabajo en el aula sobre las distintas formas de reciclaje e incluso la charla de un experto en la temática. Además, se puede visitar el entorno y participar en el trabajo que se realiza en un basurero, y reflexionar sobre la relación de estos con los cementerios en cuanto a la localización geográfica dentro de los núcleos de población. Podría ser interesante para los alumnos realizar una entrevista a un profesional con el objetivo de eliminar tabúes y prejuicios hacia una profesión que trabaja con lo inutilizable y lo desechable.

Tercera actividad: «Recuperación de la memoria histórica»

Etapa: Secundaria o Estudios Superiores (adaptable).

Objetivos:

- Generar en el alumnado responsabilidad socio-histórica y humanitaria.

- Reflexionar sobre la importancia de contemplar el pasado con el objetivo de mejorar y participar en la evolución de las sociedades.

- Indagar en experiencias de personas que han sacrificado su vida por causas humanitarias.

Contenidos:

- Memoria histórica.

- Experiencias vitales de personas comprometidas.

- Responsabilidad socio-histórica. 
Contribución a las competencias básicas:

- Competencia en comunicación lingüística.

- Competencia social y ciudadana.

- Competencia en el conocimiento y en la interacción con el mundo físico.

- Competencia cultural y artística.

Áreas curriculares vinculadas:

- Ciencias sociales, geografía e historia (Secundaria).

- Educación para la ciudadanía y los derechos humanos (Secundaria).

- Lengua castellana y literatura (Secundaria).

- Educación ético-cívica (Secundaria).

Sugerencias para su realización: En una sociedad generalmente más preocupada por el bienestar individual, la Pedagogía de la Muerte puede contemplar entre sus finalidades el desarrollo de la responsabilidad sociohistórica. La recuperación de la memoria histórica contiene elementos que pueden ser trabajados tanto en el aula como a través de la participación y colaboración con instituciones externas al centro. Se sugiere, por otra parte, la comunicación inter-generacional como medio para generar sensibilidad y conciencia diacrónica. En este sentido, las actividades pueden integrar entrevistas con personas mayores, estudio y difusión social de personas que han sacrificado sus vidas por diferentes causas o excursiones a fosas comunes a través de la colaboración con fundaciones o entidades sin ánimo de lucro.

Cuarta actividad: "Causas solidarias y humanitarias ante desastres o guerras»

Etapa: Infantil, Primaria, Secundaria o Estudios Superiores (adaptable).

Objetivos:

- Favorecer la sensibilidad hacia desastres o guerras, aun afectando en zonas geográficas lejanas. 
- Generar una identidad universal basada en la identificación con el otro, la empatía y la compasión.

- Compensar la desigualdad que suelen dar los medios de comunicación a las muertes en función de la nacionalidad del muerto, su posición social y económica o su popularidad.

\section{Contenidos:}

- Conocimiento de desastres naturales o guerras.

- Actitudes críticas hacia el tratamiento en los medios de comunicación de los desastres naturales o las guerras.

- Jerarquía de muertes.

- Empatía y sensibilidad.

Contribución a las competencias básicas:

- Competencia social y ciudadana.

- Competencia en el conocimiento y en la interacción con el mundo físico.

Áreas curriculares vinculadas:

- Conocimiento de sí mismo y autonomía personal (Infantil).

- Conocimiento del entorno (Infantil).

- Conocimiento del medio natural, social y cultural (Primaria).

— Ciencias sociales, geografía e historia (Secundaria).

- Educación ético-cívica (Secundaria).

- Educación para la ciudadanía y los derechos humanos (Primaria y Secundaria).

Sugerencias para su realización: Uno de los objetivos de la Pedagogía de la Muerte es que los estudiantes entiendan al ser humano desde su esencia y no en función de su nacionalidad, posición social y económica o popularidad. Esta desigualdad, en parte, es causa de la jerarquía de muertes que 
nos suelen presentar los medios de comunicación, ya que al tratar desastres naturales o guerras se fundamentan más en el morbo y en el ego cultural, local o nacional que en la identificación de la persona con la humanidad a través de la empatía, la compasión y el respeto. A través del trabajo en el aula en áreas curriculares como las señaladas y de acciones sociales como la recaudación de ropa o juguetes, la realización de conciertos solidarios, etc. la educación puede compensar el desajuste presentado generalmente en los medios de comunicación.

Quinta actividad: "Participación en un programa de radio local para explicar los rituales funerarios en las diferentes culturas que nos encontramos en el centro educativo»

Etapa: Primaria o Secundaria (adaptable).

Objetivos:

- Fomentar el respeto a la diversidad de culturas que coexisten en un centro educativo.

- Normalizar temas cercanos a la muerte que suelen ser tabú en la sociedad y en las instituciones educativas.

- Favorecer la relación entre medios de comunicación y centros educativos como dialéctica imprescindible para la formación personal.

Contenidos:

- Conocimiento del funcionamiento de una emisora de radio.

- Respeto a la diversidad.

- Rituales funerarios en diferentes culturas.

Contribución a las competencias básicas:

- Competencia social y ciudadana.

- Competencia en el conocimiento y en la interacción con el mundo físico.

- Competencia en comunicación lingüística. 
- Competencia cultural y artística.

- Tratamiento de la información y competencia digital.

Áreas curriculares vinculadas:

— Conocimiento del medio natural, social y cultural (Primaria).

- Ciencias sociales, geografía e historia (Secundaria).

- Educación ético-cívica (Secundaria).

— Lengua y cultura castellana (Primaria y Secundaria).

- Educación para la ciudadanía y los derechos humanos (Primaria y Secundaria).

Sugerencias para su realización: La participación de los medios de comunicación en la formación es un elemento básico para la mejora de la calidad de la educación. Los centros educativos, en este sentido, tienen que hacer un esfuerzo por colaborar con estas instituciones. Los alumnos pueden, por otra parte, ejercer su responsabilidad y realizar un servicio a la comunidad a través de la participación en estos medios de comunicación. Esta actividad global puede integrar propuestas didácticas para trabajar en el aula como el conocimiento del funcionamiento de las emisoras de radio o el estudio de los rituales funerarios en diferentes culturas, aprovechando la diversidad que se encuentra actualmente en los centros educativos. Se pueden aprovechar días señalados como el día de los difuntos, y colaborar con emisoras de radio locales para participar en un programa sobre los rituales funerarios en diferentes culturas. Es muy importante, por tanto, la coordinación de los profesionales del centro educativo con los medios de comunicación que van a participar.

Sexta actividad: "Apoyo a personas mayores y reorientación de los valores vitales»

Etapa: Secundaria o Estudios Superiores (adaptable).

Objetivos:

- Favorecer el diálogo y la comunicación inter-generacional.

- Fomentar el respeto a personas mayores. 
- Tener conciencia del ciclo de la vida y de la finitud del ser humano como condición para reorientar los valores vitales.

\section{Contenidos:}

- Respeto y tolerancia.

- Ciclo vital.

- Valores vitales.

Contribución a las competencias básicas:

- Competencia social y ciudadana.

- Competencia en comunicación lingüística.

- Competencia en el conocimiento y en la interacción con el mundo físico.

Áreas curriculares vinculadas:

- Ciencias sociales, geografía e historia (Secundaria).

- Ciencias de la naturaleza (Secundaria).

- Educación para la ciudadanía y los derechos humanos (Secundaria).

- Educación ético-cívica (Secundaria).

Sugerencias para su realización: Las instituciones educativas pueden ayudar a que se recupere la comunicación inter-generacional y se respete a las personas mayores por su importante contribución pasada y actual a la sociedad y al conocimiento. La actividad puede abarcar charlas de profesionales que trabajan con personas ancianas en el centro educativo, apoyo del alumnado en tareas en una residencia de ancianos o en otro tipo de centros de apoyo a estas personas, o reflexión posterior en el aula acerca de la experiencia. En este sentido, pueden guiar la reflexión personal y grupal sobre la experiencia preguntas como las siguientes: ¿cómo te imaginas de anciano o anciana? ¿Qué te gustaría pensar en esos momentos? ¿Cómo te gustaría que hubiera sido tu vida? ¿Hay cosas en la vida que valores más al pensar que algún día puedes llegar a ser anciano y morir? 


\section{CONCLUSIONES}

La muerte, generalmente apartada de la educación y normalmente evitada por la Pedagogía, puede vincularse a valores y sentimientos como la generosidad, la conciencia social o la solidaridad. La Pedagogía de la Muerte ahonda en lo más profundo, interior y orientador de la persona. La educación, a través de propuestas metodológicas como el AS, puede contribuir a la mejora de las futuras generaciones y cultivar en cada persona lo que le es propio de su condición humana. Consideramos que ninguna metodología didáctica es educativa a priori. Para serlo su dimensión teleológica o su sentido formativo han de estar presentes en su desarrollo. Es el valor formativo de las actividades didácticas que se llevan a cabo lo que justifica el uso de una metodología, y a nuestro entender la muerte, normalizada y tratada de forma natural, puede ser un elemento pedagógico clave para la evolución humana. Con esta pretensión la formación inicial y continua del profesorado resulta un aspecto fundamental. Desde esta perspectiva pedagógica, el AS como metodología relevante para el desarrollo de la Pedagogía de la Muerte, puede fomentar una mayor conciencia desde la que ejercer de forma más madura la responsabilidad, la sensibilidad y la acción solidaria. Como puede apreciarse en las propuestas didácticas planteadas, la muerte y los conceptos que la rodean y amplían su percepción desde un enfoque pedagógico - muerte parcial, ciclo vital, muerte continua, reorientación de los valores, transculturalidad, etc. (Herrán y Cortina, 2006) - está presente en la vida cotidiana de una comunidad y por ello puede incluirse en las acciones formativas a través de metodologías didácticas que favorezcan la intervención social y solidaria de los alumnos y alumnas. Dichas propuestas son experiencias didácticas que actualmente se están desarrollando y definen rutas relativamente novedosas para un desarrollo pedagógico formativo. 


\section{REFERENCIAS BIBLIOGRÁFICAS}

Ariés, P. (2000). Historia de la muerte en occidente: desde la edad media hasta nuestros días. Madrid: Acantilado.

Bowie, L. (2000). Is there a place for death education in the primary curriculum? Pastoral Care, 1, 22-26.

Cabrera, J. (2011). Creatividad, conciencia y complejidad: una contribución a la epistemología de la creatividad para la formación. Tesis doctoral, Universidad Autónoma de Madrid.

Collins, S. (2009). Los juegos del hambre. Barcelona: RBA Libros.

Cortina, M. (2010). El cine como recurso didáctico de educación para la muerte: implicaciones formativas para el profesorado. Tesis doctoral, Universidad Autónoma de Madrid.

Damasio, A. (2010). Antonio Damasio: El Mago del Cerebro [Entrevista]. El País Digital (10/11/2010).

Dürkheim, K. G. (1996). El maestro interior. Bilbao: Mensajero.

Gorer, G. (1995). The pornography of death. Encounter, Oct., 49-52.

Herrán, A. de la (1998). La conciencia humana. Hacia una educación transpersonal. Madrid: San Pablo.

Herrán, A. de la (2005). El nuevo paradigma complejo-evolucionista en educación, en A. de la Herrán, E. Hashimoto y E. Machado (Coords.), Investigar en educación. Fundamentos, aplicación y nuevas perspectivas (pp. 481-571). Madrid: Dilex.

Herrán. A. de la (2006). Los estados de conciencia: análisis de un constructo clave para un enfoque transpersonal de la didáctica y la formación del profesorado. Tendencias Pedagógicas, 11, 103-154.
Herrán, A. de la (2008). Hacia una Educación para la Universalidad: Más allá de los ismos, en J. Valle (Coord.), De la identidad local a la ciudadanía universal: el gran reto de la educación contemporánea (pp. 209-257). Bilbao: Fundación para la Libertad-Universidad Autónoma de Madrid.

Herrán, A. de la (2012). Fundamentos para una Pedagogía de la Muerte, en M. Cortina y A. de la Herrán (Coords.), Pedagogía de la Muerte a través del cine (pp. 23-126). Madrid: Universitas.

Herrán, A. de la y Cortina, M. (2006). La muerte y su didáctica. Manual para Educación Infantil, Primaria y Secundaria. Madrid: Humanitas.

Herrán, A. de la y Cortina, M. (2008). La Práctica del 'Acompañamiento Educativo' desde la Tutoría en Situaciones de Duelo. Tendencias Pedagógicas, 13, 157-173.

Herrán, A. de la, González, I., Navarro, M. J., Freire, V. y Bravo, S. (2000). ¿Todos los caracoles se mueren siempre? Cómo tratar la muerte en Educación Infantil. Madrid: Ediciones de la Torre.

Herrán, A. de la, González, I., Navarro, M. J., Bravo, S. y Freire, M. V. (2001). La Muerte: ¿Tabú o Imperativo Educativo? Aula de Innovación Educativa, 106, 62-64.

Herrán, A. de la y Muñoz, J. (2002). Educación para la universalidad. Más allá de la globalización. Madrid: Dilex.

Herrán, A. de la (2014). Enfoque radical e inclusivo de la formación. REICE. Revista Electrónica Iberoamericana sobre Calidad, Eficacia y Cambio en Educación, 12(2), 163-264. Recupe- 
rado de: http://www.rinace.net/reice/ numeros/arts/vol12num2/art8.pdf.

Jacoby, B. (2003). Building partnerships for service-learning. San Francisco: Jossey-Bass.

Mantegazza, R. (2006). La muerte sin máscara: experiencia del morir y educación para la despedida. Barcelona: Herder.

Montaigne, M. de (2003). Ensayos completos. Barcelona: Ediciones Cátedra.

Nietzsche, F. (1980). Ecce homo. Cómo se llega a ser lo que se es. Madrid: Alianza Editorial.

Nietzsche, F. (1984). La gaya ciencia. Barcelona: Calamus Scriptorius.

Puig, J., Batlle, R., Bosch, C. y Palos, J. (2006). Aprendizaje servicio. Educar para la ciudadanía. Madrid: Octaedro.

Rodríguez Herrero, P. (2012). Pedagogía de la Muerte en personas con discapacidad intelectual. Elaboración, aplicación y evaluación de un programa educativo. Tesis Doctoral, Universidad Autónoma de Madrid.

Rodríguez Herrero, P., Herrán, A. de la y Cortina, M. (2012). Antecedentes de Pedagogía de la Muerte en España. Enseñanza \& Teaching, 30(2), 175-195.

Rodríguez Herrero, P., Herrán, A. de la e Izuzquiza, D. (2013). "Y si me muero... ¿dónde está mi futuro?» Hacia una educación para la muerte en personas con discapacidad intelec- tual. Educación XX1 (16.1), 329-350. DOI: 10. 5944/educxx1.16.1.729.

Rubio, L. y Arroyes, A. (2010). La ecoauditoría en el aula, en X. Martín y L. Rubio (Coords.) Prácticas de ciudadanía. Diez experiencias de aprendizaje servicio (pp. 65-83). Madrid: Octaedro.

Rubio, L. y Escardíbul, S. (2010). Todos somos meninos, en X. Martín y L. Rubio (Coords.) Prácticas de ciudadanía. Diez experiencias de aprendizaje servicio (pp. 157-171). Madrid: Octaedro.

Savater, F. (1997). El valor de educar. México: Ediciones del Instituto de Estudios Educativos y Sindicales de América.

Tapia, M. N. (2004). Aprendizaje y servicio solidario. Madrid: CCS-ICCE.

Tapia, M. N. (2008). Calidad académica y responsabilidad social: el aprendizaje servicio como puente entre dos culturas universitarias, en M. Martínez (Ed.) Aprendizaje servicio y responsabilidad social de las universidades (pp. 27-56). Madrid: Octaedro.

Torre, S. de la (2006). Creatividad en educación, en S. de la Torre y V. Violant (Coords.) Comprender y evaluar la creatividad, Vol. I. (pp. 123-154). Málaga: Aljibe.

Titlebaum, P., Williamson, G., Daprano, C., Baer, J. y Brahler, J. (2004). Annotated history of service-learning. Dayton, Ohio: University of Dayton.

\section{RECURSOS CINEMATOGRÁFICOS}

Resnais, A. (1955). Noche y niebla. Francia: Argos Films. 


\section{PERFIL ACADÉMICO Y PROFESIONAL DE LOS AUTORES}

Pablo Rodríguez Herrero, Doctor en Educación. Máster en Calidad y Mejora de la Educación. Diploma en Estudios Avanzados en Creatividad Aplicada. Ha publicado artículos, capítulos de libro y comunicaciones en temas como pedagogía de la muerte, calidad de vida en personas con discapacidad intelectual, creatividad aplicada a la educación y formación de profesores.

Agustín de la Herrán Gascón, Doctor en Educación. Profesor titular de Didáctica y Organización Escolar de Universidad. Departamento de Didáctica y Teoría de la Educación. Facultad de Formación de Profesorado y Educación. Universidad Autónoma de Madrid. www.uam.es/agustin.delaherran.

Mar Cortina Selva, Doctora en Educación. Durante diez años impartió cursos sobre acompañamiento a las personas que van a morir y formación de voluntariado social. Actualmente imparte cursos sobre los niños y la muerte y sobre Educación. Preside la Asociación Española de Tanatología. Es autora del cuento infantil «On és el iaio?» publicado por Tandem en 2001 y del Manual «La muerte y su didáctica», publicado por Universitas en 2006, así como diferentes artículos sobre temas educativos.

Dirección de los autores: Agustín de la Herrán Gascón

Departamento de Didáctica y Teoría

de la Educación

Universidad Autónoma de Madrid

C/ Francisco Tomás y Valiente, 3

28049 Madrid

E-mail: agustin.delaherran@uam.es

Fecha Recepción del Artículo: 06. Junio. 2012

Fecha Modificación Artículo: 23. Agosto. 2012

Fecha Aceptación del Artículo: 28. Septiembre. 2012

Fecha Revisión para publicación: 15. Mayo. 2014 
